\title{
Monitoring Inkjet Printer Condition via Image Analysis of Printed Patterns
}

\author{
Christoph Zeh ${ }^{1}$, Peter Bischoff', Marek Rjelka ${ }^{1}$, Christiane Schuster ${ }^{1}$, Thomas Härtling ${ }^{1,2}$ \\ ${ }^{1}$ Fraunhofer Institute for Ceramic Technologies and Systems, Maria-Reiche-Str. 2, \\ 01109 Dresden Germany \\ 2 Technische Universität Dresden, Institute of Solid State Electronics, Mommsenstraße 15, \\ 01069 Dresden, Germany \\ christoph.zeh@ikts.fraunhofer.de
}

\begin{abstract}
Summary:
Detailed information on the condition of large-character inkjet printers can be acquired by using intermediate results from standardized matrix code grading. This approach allows monitoring each individual printer nozzle which is of high importance if ceramic inks are used for printing. Inks containing ceramic pigments tend to clog printer nozzles more rapidly which may lead to loss of print quality. In several application areas such as hot metal forming code quality must be guaranteed which can be achieved with the approach reported here.
\end{abstract}

Keywords: direct part marking, data matrix code, grading, condition monitoring, image analysis

\section{Introduction}

Direct Part Marking (DPM) summarizes a variety of technologies to provide digital codes directly onto products or individual components. Each part receives a unique identifier (UID). UIDs and subsequent tracking and tracing of parts are key to industrial digitalization (Industry 4.0). Here, we show how existing code grading standards can be expanded for continuous monitoring of individual printer nozzles and how this provides in-depth information on the printer condition and possible causes for malfunctions.
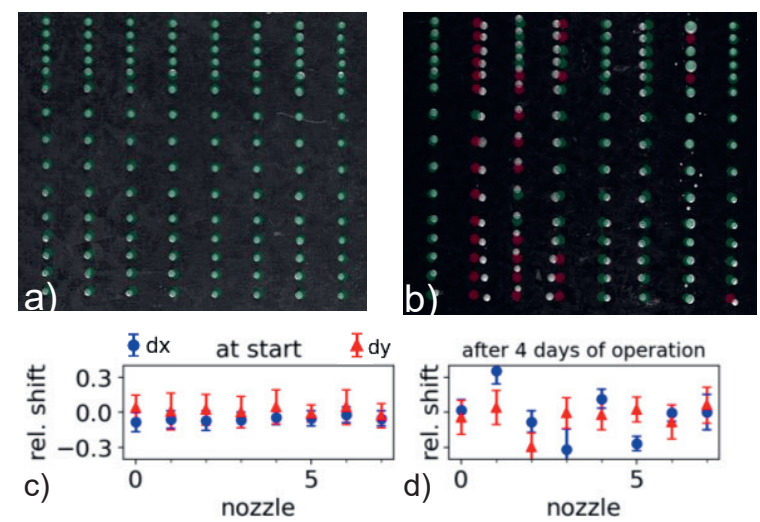

Fig. 1. Printed test-patterns a) initially and b) after 4 days of continuous operation, respectively. Color overlay indicates brightness grading. Corresponding$l y, c)$ and d) show mean and SD of position deviations.

\section{Background, Motivation and Objective}

Typically, Data Matrix ECC200 or QR codes are chosen to encode UIDs. The fastest DPM- technology in terms of coded parts per time unit is inkjet printing. When selected solid particulate materials (e.g. ceramic pigments) are brought onto the parts via the ink, hightemperature processes like hot forming (metal industry) can be addressed by inkjet technology. A drawback of using non-standard pigment materials and larger particle sizes in the ink formulations is that they can only be applied through rather coarse inject nozzles ( $\geq 100 \mu \mathrm{m})$. Suitable large-character printers are based on electro-magnetic valve technology and typically contain 16 nozzles or multiples thereof. Compared to other printing technologies, larger nozzles cause a significant increase in drop volumes and hence larger drop diameters on the destined substrate surfaces. Since the available area is limited on most product components, there is an upper limit to the matrix code dimensions and consequently a limit on the number of drops that can be used in each dimension (resolution). This means each single drop applied to the component strongly impacts the quality and readability of the resulting matrix codes. Malfunctioning of a single printer nozzle can lead to total quality loss (according to industry standards) and often, but not necessarily, to loss of decodability. Therefore, monitoring the condition of printers is of paramount importance to ensure continuous operation. Existing standards for grading matrix codes provide rules to grade and verify DPM code quality via optical inspection and image analysis. Verifying every single DPM code is common practice in 
the industry, so imaging equipment is easily available and could be exploited for further analysis. However, common standards only allow for an overall print quality measure and give limited information on the printer's current condition. In particular, single nozzle malfunctions cannot be measured despite that they have to be avoided and, if possible, predicted long before the print quality is drastically reduced. Therefore, the goal of this work is to define those measures that describe the condition as completely and exact as possible only using image analysis similar to existing standards.

\section{Monitoring gradual nozzle clogging via im- age analysis}

The most common malfunction for inkjet printers is nozzle clogging. This can occur stochastically, going from normal operation to total nozzle blocking in a single operation if large particle agglomerates get stuck in a nozzle. But clogging can also gradually build up through continuous growth of small residue. While the first form of error is hard to predict, the second form of error should be visible in specific features of the printed codes. Gradual clogging will reduce the diameter of the nozzle and hence reduce drop volume. Although, small residue may not hinder ink flow significantly, but rather only lead to subtle changes in the drop flight direction, leading to a change in drop position with respect to the desired position.

ISO 15415 (with ISO TR 29158) provides rules to measure brightness, contrast, and modulation for each cell of a matrix code as interim results before overall grades are determined. Physically, these features mainly correspond to the lateral pigment distribution and their optical properties. Varying surface characteristics of the components, which provide the image background, shall be ignored at this point. Provided the orientation of the printer nozzles with respect to the matrix code is known, brightness and similar features can be attributed to a particular nozzle by selecting the corresponding cells of the matrix code. Via brightness, gradual nozzle clogging is detectable due to a reduction of pigments and due to shift of drop position with respect to the matrix grid which in turn translates to brightness changes at a specific matrix location. Slight positional changes might go unnoticed with this approach (Fig. $1 \mathrm{a}, \mathrm{b}$ ).

EN 9132 takes position and size of each element in the code matrix into account. These measures relate more directly to the impact of gradual nozzle clogging if intermediate grading results are used to gain nozzle-wise information (Fig. 1 c, d). Noteworthy, drop size measurements can be misleading if changes in bright- ness or varying substrate conditions are not considered simultaneously. Moreover, drop sizes strongly depend on threshold values to separate foreground and background of the image and can be difficult to interpret if the lateral particle distribution is inhomogeneous. In these cases, Al-driven approaches for segmentation can provide much more reliable results than the purely analytical analysis defined in the grading standards mentioned above. Some of these will be presented in another contribution.

\section{Separating nozzle clogging from other printer malfunctions}

Some relevant causes for print quality degradation are ink pressure variations, filter clogging, abrasion, and temperature or humidity changes. Their effects should apply similarly to all printer nozzles. For example, all drop sizes of one printed code change following a slow pressure change, or the brightness of all dots are reduced in case of filter clogging which reduces the ink's solid particle concentration. These effects can be separated from nozzle clogging by comparing mean measures of each nozzle with the overall mean of the matrix code. Temperature has a strong impact on ink viscosity and therefore affects drop break-off from the nozzle and build-up of residues at the nozzle exit. This process will be more subtle, and the size of the effect will differ for each nozzle since it also depends on the latest history of the printer. Therefore, these effects are better described by changes of variance of the quality measures. All measurements introduced above need to be carried out for each printed matrix code and monitored over time. Only then, degradation of the printer condition can be evaluated in a useful manner.

\section{Conclusion and Outlook}

In summary, a detailed evaluation of the condition of large-character inkjet printers can be acquired by using intermediate results from existing matrix code grading standards and keeping track of nozzle-correspondence. In the future, the described measures will be used to build robust machine learning models that allow to predict nozzle clogging and other printer malfunctions for predictive and automated printer maintenance.

\section{References}

[1] ISO/IEC 15415:2011, Information technology Automatic identification and data capture techniques - Bar code symbol print quality test specification - Two-dimensional symbols

[2] EN 9132 Aerospace series - Quality management systems - Data Matrix Quality Requirements for Parts Marking 